\title{
Overview on initial METIS D2D Concept
}

\author{
(Invited Paper)
}

\author{
Zexian Li, Martti Moisio, Mikko A. Uusitalo, Petteri \\ Lundén, Carl Wijting \\ Nokia Technologies \\ Espoo, Finland \\ E-mail: firstname.middleinitials.lastname@nokia.com
}

\author{
Fernando Sanchez Moya, Ali Yaver, Venkatkumar \\ Venkatasubramanian \\ Nokia Networks \\ Wrocław, Poland
}

\begin{abstract}
METIS is the EU flagship project with the purpose of developing a $5 \mathrm{G}$ system concept that meets the requirements of the beyond-2020 connected information society and emerging applications. Within METIS project, horizontal topics (HTs) are used to build the overall system concept. Direct Device-to-Device (D2D) communication is one of the HTs and regarded as a promising technology to provide low power, high data rate and low latency services between end-users in the future $5 \mathrm{G}$ networks. This paper presents an initial view on the HT D2D concept. In addition, the performance of selected technology components is included in this paper as well.
\end{abstract}

Keywords- 5G; Direct Device-to-Device (D2D) communication; METIS; HT; System concept;

\section{INTRODUCTION}

The overall objective of METIS (Mobile and Wireless Communications Enablers for the Twenty-Twenty Information Society) is to develop a $5 \mathrm{G}$ system concept that meets the requirements of the beyond-2020 connected information society and broadens the use of today's cellular communication systems to support new usage scenarios [1]. The developed concept should support the METIS overall technical goals:

- 1000 times higher mobile data volume per area,

- 10 to 100 times higher number of connected devices,

- 10 to 100 times higher user data rate,

- 10 times longer battery life for low power massive machine communication (MMC), and

- 5 times reduced End-to-End latency.

These requirements shall be achieved at a similar cost and energy consumption level as today. In order to derive the requirements and key performance indicators (KPIs), METIS has analyzed the identified scenarios and applications. As a consequence, the requirements of the connected information society are captured in five METIS scenarios [1].

METIS selected five Horizontal Topics (HTs) to facilitate the system concept development: Direct Device-to-Device (D2D) Communication, Massive Machine Communication (MMC), Moving Networks (MN), Ultra-Dense Networks (UDN), and Ultra-Reliable Communication (URC). Each HT addresses certain new challenges derived from the identified scenarios and test cases, identifies necessary new functionalities

This work is performed as a part of the EU FP7 project METIS, ICT317669 which is partly funded by the EU. and develops HT concept by integrating the Technology Components studied in technical Working Packages (WPs).

Direct D2D communication, which refers to networkcontrolled direct communication between devices without Core Network (CN) involvement in the user-plane of local communication, has been seen as one of the most important elements in improving system performance and supporting new services/applications for beyond 2020. The HT D2D concept addresses the challenges related to local information exchange among the devices and creates a framework for solving these technical challenges. HT D2D can contribute to METIS technical goals including at least increased user data rate, increased number of connected devices and reduced latency in various aspects via for example traffic offloading and local information exchange among devices.

The rest of the paper is organized as follows. In Section II we give an introduction of the METIS D2D and related technical challenges. In Section III we present an initial view of the corresponding METIS D2D concept. Section IV shows the performance of example D2D technology components and finally Section $\mathrm{V}$ concludes the paper.

\section{D2D AND RELATED TECHNICAL CHALLENGES}

\section{A. Overview of METIS D2D}

Network assisted D2D communication enables direct communication between devices in proximity. METIS aims to naturally integrate direct D2D communication as part of the overall communication system. The objectives of METIS D2D include increasing coverage (availability and reliability), offloading backhaul (cost efficiency), providing a fall back solution (reliability), improving spectrum usage (spectrum efficiency), typical user data rate and capacity per area (capacity density), and enabling new services and experiences for example V2X communication (including at least communication between vehicles - V2V (Vehicle to Vehicle) services and V2I (Vehicle to Infrastructure) services) as described in [1].

Network controlled direct D2D communication offers the opportunity for local management of short-distance communication links, which allows separation of local traffic from the global network (i.e. local traffic offloading). This will not only significantly ease the load on the backhaul and the $\mathrm{CN}$ 
which is caused by data transfer and related control signaling, but will also reduce the necessary effort for traffic management at the central network nodes. Based on direct D2D communication, local data caching and sharing zones can be easily set up, allowing content sharing by a large number of users without putting heavy load on the wide area network. Direct D2D communication therefore extends the idea of distributed network management by incorporating the end devices themselves into the network management concept. In another flavor of direct D2D communication, mobile devices may act as relay stations in a multi-hop transmission environment from distant mobiles to base stations. These cases considered within METIS are illustrated in Figure 1.

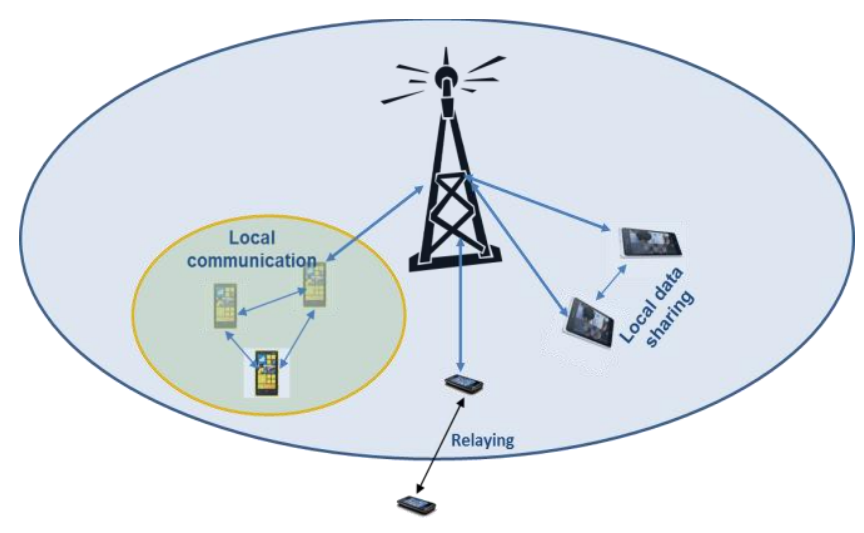

Figure 1 Exemplary usage of direct D2D communication scenarios

Direct D2D communication can be utilized in various scenarios with significantly different requirements and thus the following sub-topics have been identified within METIS [2]:

- D2D-N for Non-critical applications, e.g. traffic offloading in mobile broadband scenarios where the service can be provided at the level of best effort.

- D2D-C for Critical/ultra-reliable applications, e.g. V2X communication, where fast establishment of the links and ultra-reliable communication of low to moderate amounts of data with very low latency are the key factors to be considered.

- D2D-M for direct M2M communication. In this case, power consumption and protocol overhead are more important than latency, and the established links can be valid for longer time due to low mobility.

- D2D-B for Backhaul applications to provide selfbackhaul in multi-hop mesh networks in e.g. UDN deployments.

\section{B. Technical challenges}

Considering the D2D relevant scenarios, the identified key technical challenges lead to at least the following questions:

- Device discovery

Efficient network assisted D2D discovery, which is used to determine the proximity between devices and the potential to establish a direct D2D link, is a key element in order to enable D2D communication and also possible new applications. Device discovery tries to solve the problem of how to enable an efficient discovery of devices in proximity. Device discovery latency and efficient resource usage are among the KPIs.

- Communication mode selection

Mode selection is another core function which controls whether two devices will communicate each other in direct D2D mode or in regular cellular mode. When the devices communicate in direct mode, they can take advantage of their proximity and may reuse cellular resources for the direct communication link. In cellular mode, the devices communicate through a common or separate serving base station by means of traditional cellular technology. How to select the most appropriate communication mode? What are the criteria for mode selection or on which time-scale should mode selection ideally be performed?

- Co-existence and interference management

Considering co-existence and related interference issues, at least two different aspects should be taken into account: (1) co-existence among a large number of D2D links, and (2) co-existence among D2D links and regular cellular links. Should we take well-known centralized interference management or distributed interference management should be adopted? In addition, the impact due to RF impairment which can bring addition interference should be studied as well.

- Multi-operator or inter-operator D2D operation

Inter-operator D2D is a clear requirement resulting from e.g. V2X communication and supporting inter-operator D2D operation is very essential to HT D2D concept. Without multi-operator D2D support, the applicability of METIS D2D solution to e.g. Cooperative Intelligent Traffic Systems (C-ITS) will be quite limited. Considering inter-operator D2D operation, issues to be solved include, for example, spectrum usage and how to control and coordinate UEs in D2D communication across multiple operators' networks.

\section{METIS HT D2D CONCEPT}

Taking into account the technical challenges as discussed in Section II, the initial METIS D2D concept is illustrated in Figure 2. From the figure it can be observed that the direct D2D communication is controlled by the network and the D2D data is shared among local devices. Within the link between local devices, it is also possible to include D2D related control information especially if considering the $\mathrm{D} 2 \mathrm{D}$ relay scenario.

As shown in Figure 2, different circles represent certain key technology components to build the HT D2D concept ranging from air interface to spectrum management. In the following, METIS D2D concept is introduced by looking at the selected key technology components to address technical challenges. 


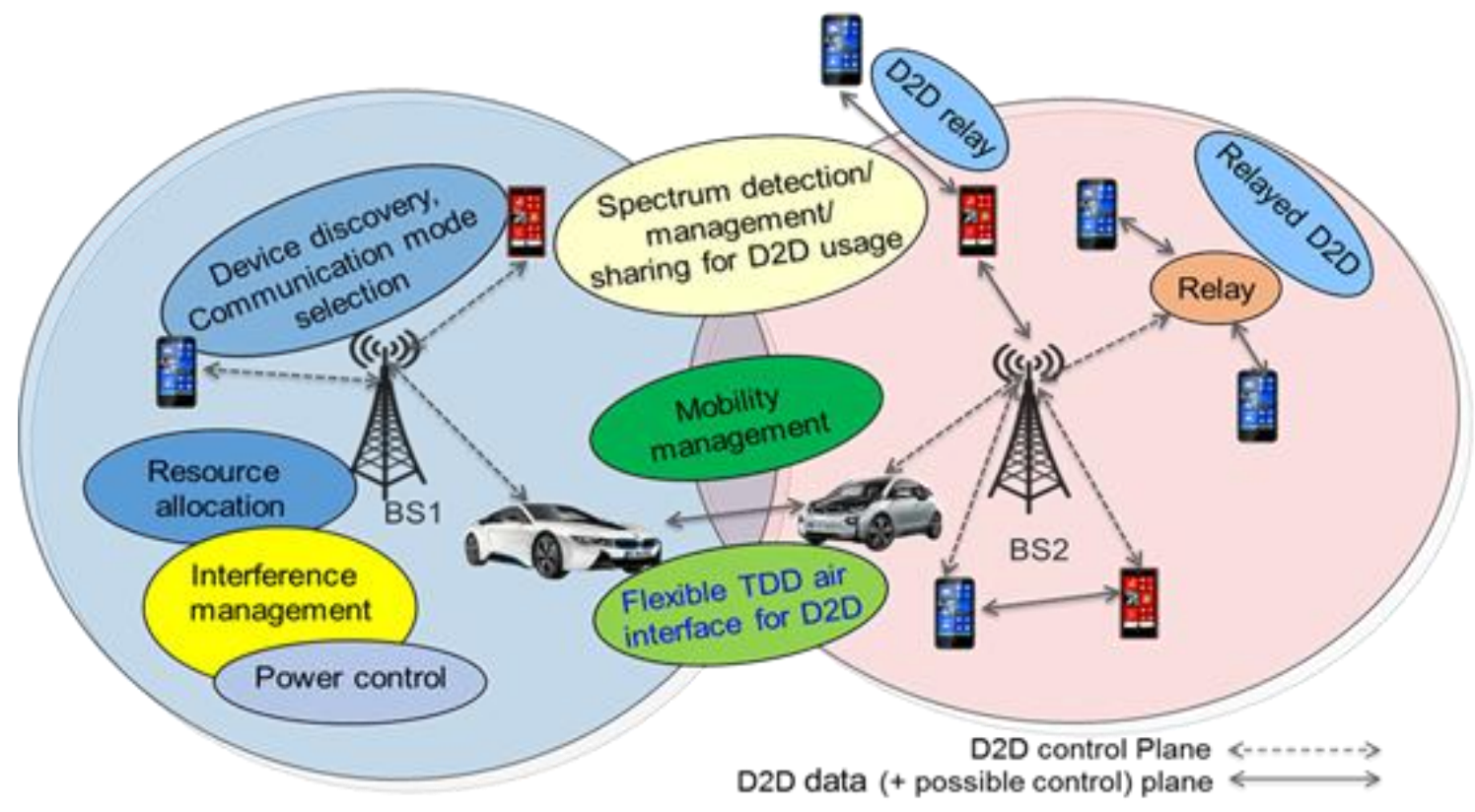

Figure 2 METIS HT D2D Concept

\section{$\underline{\text { Flexible air interface }}$}

As a result of the broad set of requirements and KPIs from 5G scenarios, a flexible air interface concept is proposed [4]. With the help of air interface management, a set of different configurations for different modes of operation are considered to allow the air interface to be individually configured according to the actual system conditions and service requests. Changing configurations should be achieved by simply adapting the parameter set. One example criterion for the air interface selection is mobility. For example if the involved D2D devices are with low mobility, a TDD based air interface composed of a scalable frame structure enables cost-efficient local communication to be selected. Ongoing research topics cover dynamic partitioning of UL/DL periods in TDD mode to account for the potential highly asymmetric traffic. However, if the devices are in the scenarios with high speed, the air interface designed for moving networks and V2X communications should be adopted. Since the quality of transmission strongly depends on the proper knowledge of the radio channel, the research embraces novel channel estimation techniques for highly timevariant channels and channel prediction. In addition, the technology component "signaling for MMC" develops an optimized signaling structure to extend the coverage of MMC via direct $\mathrm{D} 2 \mathrm{D}$ communication.

\section{- Device discovery}

A unified discovery framework is under investigation by taking into account the benefits from both centralized and decentralized solutions, i.e. both UE-based and NW-based schemes. Methods for adjusting the size of the resource pool (used for discovery message transmission) and grouping UEs to achieve high resource reuse are investigated to increase the discovery efficiency. The trade-off between the centralized and decentralized mechanisms is evaluated by means of discovery probability. Cluster based discovery scheme is under study which is more suitable for the scenarios with partial or no NW coverage.

\section{- Communication mode selection}

With the assumption that D2D is operated with the same carrier frequency as regular cellular communication, both distributed Channel State Information (CSI) based and location based mode selection schemes are studied.

- Distributed CSI-based mode selection: depending only on the information that is specific to the $\mathrm{D} 2 \mathrm{D}$ pair. With the assumption that the large scale fading between the D2D transmitter and receiver and between the D2D transmitter and the Base Station (BS) are available, the direct D2D communication mode is selected if the hypothetical capacity values corresponding to the direct links are higher than the hypothetical capacity values of the cellular links, being possible to apply an offset $\Delta$ value (a configurable system parameter measured in $\mathrm{bit} / \mathrm{s} / \mathrm{Hz}$ ) to control the offloading of the infrastructure by direct D2D communication.

In addition, mode selection in overlay D2D can take advantage of the fact that only D2D users transmit in the D2D part of the spectrum. A potential D2D user measures the D2D spectrum and uses a threshold-based test (e.g. energy detection) to decide whether it transmits in D2D mode or in cellular mode. In this way, the mode selection becomes completely decentralized eliminating signaling overhead between the D2D users and BS. And mode selection for the scenario with UEs from different operators is considered as well. 
- Location based mode selection: based on users' location information, the BS estimates the distances between candidate D2D users and between each of the candidate users and the BS. The distances are then mapped to the corresponding received signal powers by utilizing appropriate path loss model. Based on the distance and the estimated path loss, the BS decides whether the users can transmit using a direct D2D link or the normal communication link via the BS.

- Combined resource allocation and mode selection in multi-cell: A flexible TDD scheme that makes use of different degrees of coordination among cells and different time scales of mode switching based on path loss or SINR is described in [5].

\section{- Co-existence and interference management}

Efficient resource allocation is one way to handle the interference issues. Within METIS, resource allocation approaches covering both single cell and multi-cell are considered. Further for location based resource allocation, the BS uses distances between the users and from the users to the BS for estimating the path losses and the Signal-to-Interference Ratio (SIR) of the users sharing the same resources. The selection of the best candidate for resource reuse is based on the distance. On top of centralized resource allocation, distributed resource allocation is considered as well, for example decentralized interference aware scheduling for D2D was studied. In addition, as another powerful tool to handle interference, distributed iterative power control is studied as well, which exploits the covariance measurements performed by D2D receivers and feeds back in a distributed fashion to the respective transmitters to adjust transmit powers. In addition, the mechanisms to handle the adjacent band interference resulting from RF impairments are studied as well. This is targeted to the scenarios where D2D and regular cellular communication are running on the adjacent carrier frequencies.

\section{- Mobility management}

Due to the introduction of direct D2D communication as a new transmission mode, an optimized mobility management procedure, e.g. by defining a new D2D handover criterion in addition to the traditional cellular handover criteria is proposed. In addition, the D2D UEs should be controlled by a minimum number of BSs in order to provide better user experience, especially considering unnecessary latency resulting from information exchange among BSs. Based on these considerations, two smart mobility management solutions were proposed [6] which can be used to minimize the negative impacts (e.g., larger latency and additional signaling overhead) of multi-site radio resource control on D2D communications by controlling the D2D handover and cell selection during the mobility of D2D UEs: D2D-aware handover and D2D-triggered handover.

\section{- D2D relay and relayed D2D}

Different forms of D2D relays are supported based on METIS solutions. For D2D relay (i.e. UE-to-NW relay), one example is the multiple-stream based relay where communication from a multi-antenna BS to a multi-antenna UE, assisted by other multi-antenna UEs acting as relay nodes, is considered. In addition, relayed D2D (i.e. UE-relay station (RS)UE) is also under investigation where two or more communication device pairs are assisted by a relay station as illustrated in Figure 2. The research topics include distributed coding for the multiple access multiple relay channel, bidirectional relay with non-orthogonal multiple access, closedloop and open-loop techniques in a network with D2D relaying. In addition, underlay D2D communication with physical layer network coding is under investigation as well [7].

\section{- Spectrum management/sharing}

Identifying suitable spectrum for D2D operation can bring significant performance improvement to the whole system. Taking into account the requirements from D2D related test cases, suitable spectrum can be identified after the analysis of pros and cons, possible ways of spectrum sharing, negotiation with other entities and other necessary steps. Moreover mechanisms enabling spectrum sharing among operators are under study as well.

\section{D2D PERFORMANCE EVALUATION}

In this section we will have a look at the system performance of two example D2D technology components: "Further enhanced Inter-Cell Interference Coordination (ICIC) in D2D enabled HetNets" and "Multi-cell coordinated and flexible mode selection and resource allocation for D2D" [5].

\section{A. Further enhanced Inter-Cell Interference Coordination in D2D Enabled HetNets}

According to the proposed scheme, the resource allocation for a direct D2D link is controlled by the macro cell and the muted subframes from macro cell for small cell usage can be re-used by D2D links if both devices are far away from the small cell. The devices of a D2D pair carry out measurements during muted subframes of controlling macro BS. If no nearby small cell detected, the D2D pair can be allocated with the resources within those muted resources, otherwise unmuted resources are used. Here we will evaluate the performance in Test Case 2 "Dense urban information society" [1]. The network layout is shown in Figure 3. Three-sector macro cells are on top of the building and two cells per micro-station point toward the main street with the same antenna pattern as macro cells. Mobiles are dropped uniformly on the streets. Detailed scenario parameters can be found in [3].

A quasi-static TDD system simulator is used. Both downlink and uplink directions are simulated at the same time during one simulation run. In the reference case, D2D is able to use macro cell UL resources only, and we compare that to the case where D2D is able to use also micro cell UL or DL resources with and without Reference Signal Received Power (RSRP) restriction (RSRP to nearest small cell). Simulation parameters are shown in Table 1 and a more detailed description of the simulation setup can be found in [2]. 


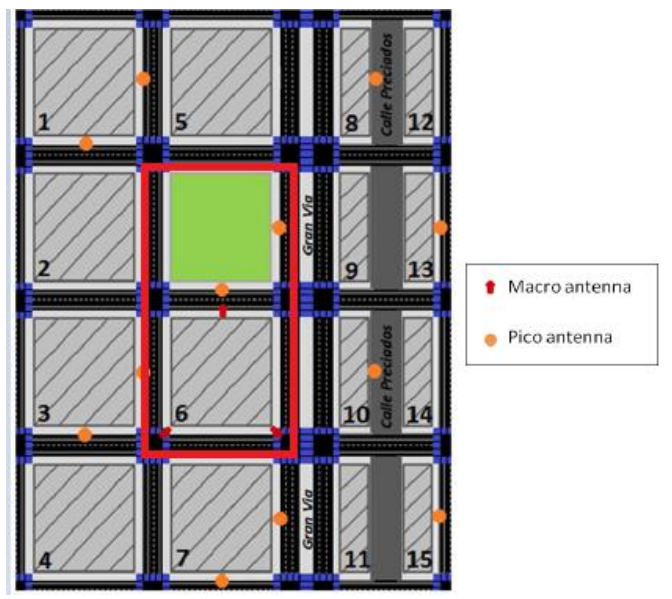

Figure 3. Network layout. The middle area in the center marked with red rectangle is the area where statistics is collected.

Table 1: Simulation Parameters

\begin{tabular}{|l|l|}
\hline Parameter & Value \\
\hline Scenario & $\begin{array}{l}\text { METIS dense urban, outdoor } \\
\text { pedestrian }\end{array}$ \\
\hline Path loss model & $\begin{array}{l}\text { METIS TC2 Outdoor and D2D } \\
\text { propagation model [3]. }\end{array}$ \\
\hline UE distribution & $\begin{array}{l}12 \text { UEs per cell, 50\% D2D, D2D } \\
\text { connections allowed up to 50m }\end{array}$ \\
\hline Spectrum & $10 \mathrm{MHz}$ at 2 GHz \\
\hline Traffic & Full Buffer \\
\hline Tx Power (Macro, pico, UE) & {$[43,30,20] \mathrm{dBm}$} \\
\hline Mobility & No \\
\hline
\end{tabular}

Figure 4 shows the gain on mean D2D UE throughput versus the corresponding loss in mean cellular UE throughput in small cells (due to the increased interference from D2D users). From the results it can be observed that the usage of the resources reserved for small cell can lead to substantial throughput/ capacity gain for D2D UEs. With properly selected threshold of the safety distance (in terms of RSRP margin from nearest small cell), the performance gain can be achieved with negligible impact on the small cell capacity. The exact gains depend on the scenario (in particular the density and coverage of the small cells). For downlink, the gains are much smaller. It should be observed that the best overall gains are achieved with quite tight RSRP restrictions for D2D communication - around $-80 \mathrm{dBm}$. These results can be used to balance the trade-off between cellular performance and D2D performance.

\section{B. Multi-cell coordinated and flexible mode selection and resource allocation for $D 2 D$}

This section introduces a joint resource allocation and mode selection scheme based on the flexible TDD-based air interface proposed in [8] and the work in [9]. The resource allocation and mode selection schemes are described as in the following:

Standalone decentralized scheme: a fully decentralized scheme without signaling exchange between small cells. However, it is assumed that each individual small cell BS gathers full information from the D2D users and the cellular users for scheduling as well as D2D reuse decisions.

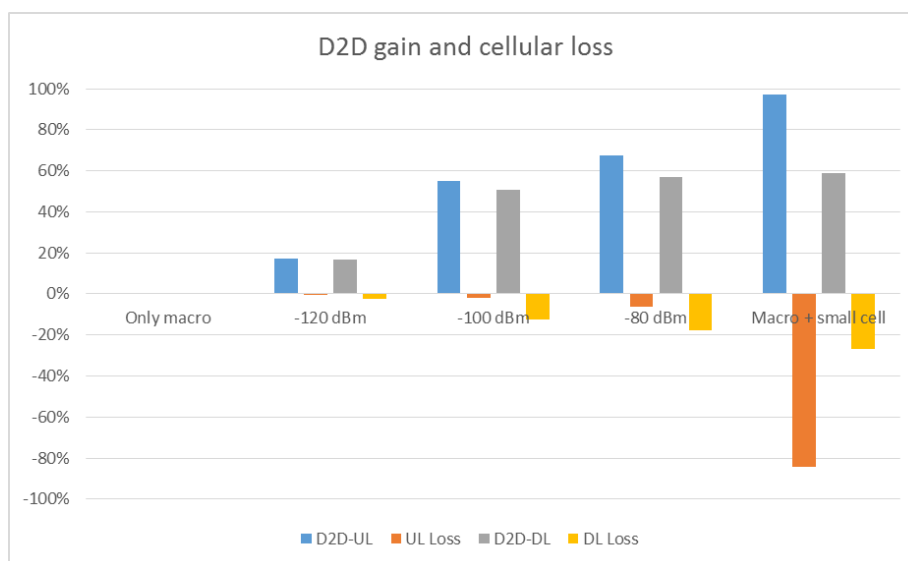

Figure 4 Gains and losses when using D2D with UL resources (D2D-UL) or with DL resources (D2D-DL).

Every BS makes resource allocation (scheduling) and UL/DL switching decisions only for its own D2D users and cellular users. In each cell scheduling decisions are made based on the criteria of packet delay weighted throughput on a resource block basis. Interference-aware scheduling is performed in slot \# $t$, by assuming that the D2D users of a small cell feedback SINR per resource block based on the estimate from time slot \# $t-1$.

Fully centralized scheme: here the resource allocation and UL/DL switching decisions are made by a central entity for both D2D and cellular users in a coordination group. In this case, it is assumed that the same entity also possesses instantaneous interference information received from all the other small cells and UEs which are not in the coordination group. Thus, scheduling decisions are made for all the links within the coordination group. The criterion is now to maximize the sum of delay weighted throughput across the D2D and cellular links in coordinating cells and is again done on a resource block basis. It is noted that muting a link is included as an option. The optimization metric is similar to [9] but now also includes D2D users in the brute-force search.

Mode selection based on path loss or slow mode selection: D2D traffic of a particular D2D pair is routed through the small cell BSs (DID, Device-Infrastructure-Device communication mode) when the uplink path loss towards the serving BS is lower than the path loss of the direct D2D link between the devices.

Mode selection based on estimated SINR from previous TTI or fast mode selection: Mode selection is based on the estimated SINR of the UL and the direct D2D link. This calculation is done on TTI level and based on SINR estimation from the previous TTI. A bias can be applied to favor direct D2D link and to leverage the hop gain over traditional DID communication.

The performance in terms of packet delay for different resource allocation and mode selection variants is compared.

The scheduler balances achievable throughput with packet delay fairness, with the key performance indicator the 95 percentile delay in terms of the serving time required between the time of packet arrival and time of the completion of packet delivery. The main simulation assumptions are summarized in Table 2. 
Results for centralized and decentralized resource management schemes together with fast and slow mode selection are shown in Figure 5. A bias to favor D2D decisions over cellular communication is applied in fast mode selection because in principle direct D2D provides a lower end-to-end delay. Resource reusing between D2D and cellular users is enabled. Gains of around 14\% in D2D packet delay reduction at 95th percentile are possible from fast mode selection over slow mode selection. The centralized scheduler performs $8 \%$ better than the decentralized one for both fast and slow mode selection cases (see green and red curves in Fig. 5). However, it sacrifices $13 \%$ of D2D packet delay for the case without mode selection (direct D2D only, see black curves in Fig. 5) while optimizing the overall system performance by achieving gains between $10 \%-16 \%$ in cellular DL and UL packet delay (not shown here due to limited space) through smart scheduling for all schemes. Note in the UE distribution and traffic parameters in Table 2 UL and DL cellular traffic have most of the load weight.

Table 2: Simulation Parameters

\begin{tabular}{|l|l|}
\hline Parameter & Value \\
\hline Scenario & $\begin{array}{l}\text { METIS dense urban, indoor only, } \\
\text { 25 cells, 10x10 m² each }\end{array}$ \\
\hline Path loss model & $\begin{array}{l}\text { 3GPP small cell path loss model } \\
\text { [10], Indoor fast fading model [11] }\end{array}$ \\
\hline UE distribution & $\begin{array}{l}\text { 12 UEs per cell (10 cellular + 2 } \\
\text { D2D), multi-cell D2D connections } \\
\text { allowed up to 16m }\end{array}$ \\
\hline Spectrum & 200 MHz at 2 GHz \\
\hline Traffic & $\begin{array}{l}\text { Poisson 1s inter-arrival time } \\
\text { DL packet size: 3.2 Mbytes } \\
\text { UL packet size: } 0.8 \text { Mbytes } \\
\text { D2D packet size: 0.8 Mbytes }\end{array}$ \\
\hline Antenna configuration & 2x2 MIMO, isotropic \\
\hline Receiver type & Interference Rejection Combining \\
\hline \multicolumn{2}{|l}{} \\
CONCLUSIONS
\end{tabular}

In this paper we introduced the METIS D2D concept which is able to provide solutions to address various technical challenges resulting from both air interface level and network level. Re-configurable radio air interface can offer the best solutions targeted to different use cases. Technology components for interference management, resource allocation, mobility management and other system level techniques enable the efficient D2D operation. We showed results of system level performance evaluation for two example D2D technology components: (1) D2D-aware ICIC scheme and (2) multi-cell coordinated and flexible mode selection and resource allocation for D2D. Substantial gains were achieved especially on D2D when micro UL resources are used with a strict RSRP safety margin. Further evaluation of the concept could, e.g., include different Quality of Service criteria for different users, like VoIP users. Finally, a D2D mode selection and resource allocation scheme based on dynamic TDD and optimized for very dense deployments was presented, showing potential benefits of centralized or coordinated resource allocation solutions along with fast mode selection. Future analysis should determine the amount of overhead and signalling needed to achieve practical gains as well as the time scale or granularity in which mode selection should operate.

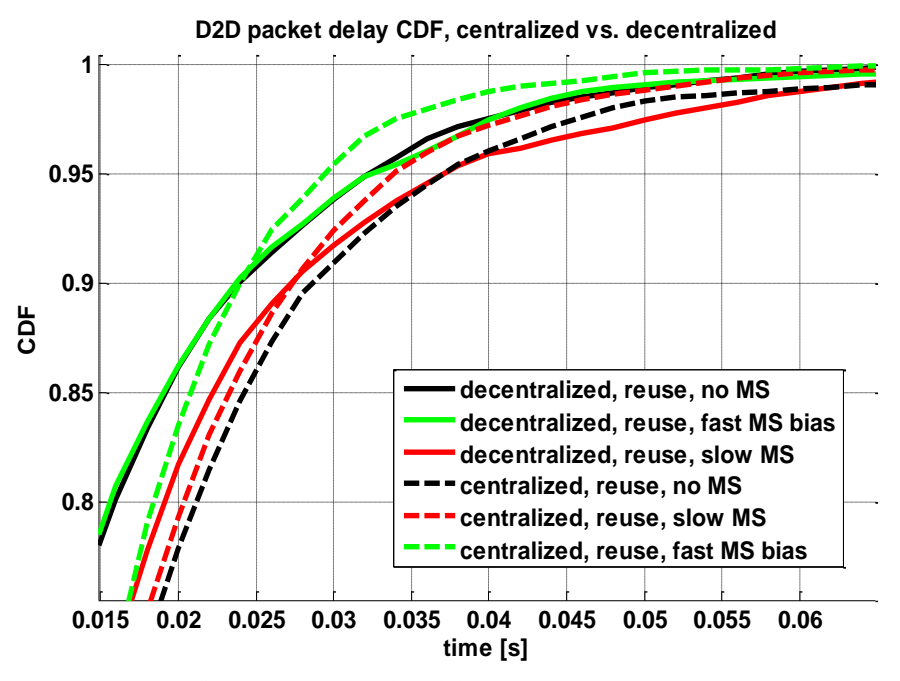

Figure 5 D2D packet delay CDF

\section{ACKNOWLEDGMENT}

The authors would like to acknowledge the work of all our METIS colleagues behind the METIS HT D2D concepts, especially the HT D2D task force members.

\section{REFERENCES}

[1] METIS Deliverable D1.1 "Scenarios, requirements and KPIs for 5G mobile and wireless system," April 2013, https://www.metis2020.com/wpcontent/uploads/deliverables/METIS D1.1_v1.pdf

[2] METIS Deliverable D6.3 "Intermediate system evaluation results," August 2014, https://www.metis2020.com/wpcontent/uploads/deliverables/METIS_D6.3_v1.pdf

[3] METIS Deliverable D6.1 "Simulation Guidelines," October 2013, https://www.metis2020.com/wpcontent/uploads/deliverables/METIS_D6.1_v1.pdf

[4] METIS Deliverable D6.2 "Initial report on horizontal topics, first results and 5G system concept", March 2014, https://www.metis2020.com/wpcontent/uploads/deliverables/METIS_D6.2_v1.pdf.

[5] METIS Deliverable D4.2 "Final report on trade-off investigations" September 2014, https://www.metis2020.com/wpcontent/uploads/deliverables/METIS D4.2_v1.pdf

[6] O. Yilmaz, Z. Li, K. Valkealahti, M. A. Uusitalo, M. Moisio, P. Lundén, C. Wijting, "Smart Mobility Management for D2D Communications in 5G Networks", WCNC, 2014.

[7] METIS Deliverable D3.2, "First performance results for multinode/multi-antenna transmission technologies"

https://www.metis2020.com/wpcontent/uploads/deliverables/METIS_D3.2_v1.pdf

[8] P. Mogensen and K. Pajukoski, "5G small cell optimized radio design," Globecom, 2013.

[9] V. Venkatasubramanian, M. Hesse, P. Marsch, M. Maternia, "On the Performance Gain of Flexible UL/DL TDD with Centralized and Decentralized Resource Allocation in Dense 5G Deployments," IEEE PIMRC 2014, Washington, USA.

[10] Proposal for Amendments to Dual-Stripe Channel Model, R1-130591, 3GPP TSG RAN WG\#1, Jan 28- Feb 1 2013, St Julian's Malta.

[11] Guidelines for Evaluation of Radio Transmission Technologies For IMT 2000, ITU-R M.1225, 1997. 